\title{
Optimizing Compliant, Model-Based Robotic Assistance to Promote Neurorehabilitation
}

\author{
Eric T. Wolbrecht, Member, IEEE, Vicky Chan, David J. Reinkensmeyer, Member, IEEE, and \\ James E. Bobrow, Member, IEEE
}

\begin{abstract}
Based on evidence from recent experiments in motor learning and neurorehabilitation, we hypothesize that three desirable features for a controller for robot-aided movement training following stroke are high mechanical compliance, the ability to assist patients in completing desired movements, and the ability to provide only the minimum assistance necessary. This paper presents a novel controller that successfully exhibits these characteristics. The controller uses a standard model-based, adaptive control approach in order to learn the patient's abilities and assist in completing movements while remaining compliant. Assistance-as-needed is achieved by adding a novel force reducing term to the adaptive control law, which decays the force output from the robot when errors in task execution are small. Several tests are presented using the upper extremity robotic therapy device named Pneu-WREX to evaluate the performance of the adaptive, "assist-as-needed" controller with people who have suffered a stroke. The results of these experiments illustrate the "slacking" behavior of human motor control: given the opportunity, the human patient will reduce his or her output, letting the robotic device do the work for it. The experiments also demonstrate how including the "assist-as-needed" modification in the controller increases participation from the motor system.
\end{abstract}

Index Terms-Assist-as-needed, motor control, nonlinear adaptive control, rehabilitation robotics.

\section{INTRODUCTION}

$\mathbf{N}$ EUROLOGICAL injury is a leading cause of permanent disability in the United States. A primary cause of neurological injury is stroke, which is suffered by over 700000 people each year [1]. About $80 \%$ of these individuals suffer a loss of control of the arm and hand. Due to the age-related risk factors of stroke, the prevalence of stroke is expected to rise as the population ages. Traditional physical therapy can enhance functional recovery after stroke [2], [3], but is labor-intensive, expensive, and likely dosage dependant [2]. In response to this problem, the

Manuscript received July 2, 2007; revised October 31, 2007; accepted December 4, 2007. This work was supported in part by the National Institutes of Health (NIH) under Grant N01-HD-3-3352 and in part by the National Center for Research Resources (NCRR) under Grant M01RR00827.

E. T. Wolbrecht is with the Mechanical Engineering Department, University of Idaho, Moscow, ID 83844 USA (e-mail: ewolbrec@uidaho.edu).

V. Chan is with the Rehabilitation Services, University of California at Irvine Medical Center, Irvine, CA 92697 USA (e-mail: vchan2@uci.edu).

D. J. Reinkensmeyer is with the Department of Mechanical and Aerospace Engineering and the Department of Biomedical Engineering, University of California, Irvine, CA 92697 USA (e-mail: dreinken@uci.edu).

J. E. Bobrow is with the Department of Mechanical and Aerospace Engineering, University of California, Irvine, CA 92697 USA (e-mail: jebobrow@ uci.edu).

Color versions of one or more of the figures in this paper are available online at http://ieeexplore.ieee.org.

Digital Object Identifier 10.1109/TNSRE.2008.918389 past two decades have seen an increase in research aimed at creating and controlling robotic devices [4]-[7] for rehabilitation following a neurological injury such as stroke. These devices attach to the limbs of patients and assist them in completing movements such as walking, reaching, and grasping.

One approach to controlling such devices is to use a relatively stiff controller to move the patient's limbs along a desired trajectory [8]. A potential problem with this approach is that using a stiff controller limits kinematic error, a key signal that drives human motor learning [9]-[11]. In addition, a stiff controller can complete the movements without active participation from the patient, which may limit the therapeutic effect of the training [12]. In order to allow some kinematic error, several devices use impedance control, notably the MIT-MANUS [13]. The compliant interaction with patients provided by impedance control better preserves the causal relationship between effort and error that is important for motor learning [14]. However, a key, unsolved problem with this approach is that it is limited in its ability to create accurate movements when it is necessary to apply large forces to a patient's limb to complete movements, such as with spatial arm motions against gravity, or situations in which the patient's limb exhibits substantial tone. More recent upper extremity devices, such as the spatial extension to MITMANUS [15] and ARMin [16] address the problem of moving the arm compliantly against gravity by adding an offset term proportional to the weight of the subject's extremity. This approach, however, does not address the configuration-dependent nature of gravitational forces or the large forces required to overcome abnormal muscle tone. Further, the amount of force a patient can contribute to a movement varies widely across patients at different impairment levels, and also within a single patient as recovery progresses and previous approaches have not systematically accounted for these variations.

Another approach to providing assistance for movement is to allow the patient to first attempt the movement, and then provide robot assistance to complete the movement, either automatically or initiated by a therapist, after a certain amount of time or lack of progress is noted, or when a desired movement falls outside of a window [17]-[21]. The goal of this "triggered" assistance approach is to provide "assistance-as-needed" by providing assistance only when the subject is unable to complete the movement, thereby increasing the muscle activity of the patient in order to encourage neural plasticity [22]. This approach was extended in [23] and [24] to include changes to controller parameters based on the previous trials. The discrete event nature of these approaches, however, requires decision either by a programmed rule set or by an observing therapist. Also, while 
simple, this approach essentially breaks the movement into two parts, a subject-driven part, and a robot-driven part, rather than providing a seamless level of robot assistance to subject-driven motion. Further, for the robot driven part, typically relatively stiff controllers have been used to achieve the necessary assistance, so that the patient is not in a compliant environment when assistance is being provided.

Initial clinical results of robotic therapy devices have shown that gains in functional recovery for patients with stroke, although modest, are significant when patients receive extra training with robotic devices [7], [25], [26]. While it remains to be demonstrated, it is possible that these gains might be increased by using more advanced robotic systems for movement training. One way to advance a robotic therapy device is to develop a controller that seamlessly optimizes the interaction between the robotic device and the recovering patient in order to provide the patient with as much therapeutic benefit as possible. Specifically, we hypothesize that a controller that maximally promotes patient involvement, while also providing enough assistance so that patients complete desired movements, will increase therapeutic benefit within the robotic assistance paradigm.

In this paper, we present a general Lyapunov-based control framework that is capable of compliantly assisting patients only "as needed" in completing reaching movements. The foundation for this control framework is an adaptive controller that learns in real time a dynamic model of the patient's arm as well as a model of the patient's ability and effort. Use of a model-based controller allows impedance and assistance to be controlled separately, so that the device can simultaneously be highly compliant and be able to provide enough assistance force to complete arbitrary spatial movements. Portions of this work have been previously published in conference paper format [27]-[30].

\section{MEthodS}

\section{A. Experimental Apparatus}

The robotic orthosis used for this work is called "PneuWREX" [27], [28] (Fig. 1). It is a 4 degrees-of-freedom robot based on a passive arm support called WREX, developed for children by [31]. WREX uses elastic bands to balance the weight the arm. Pneu-WREX is a larger version of WREX that uses a spring to balance its own weight, and incorporates pneumatic actuators to generate active forces. The development of the force controller for the pneumatic controller is described in [29]. Essentially, Pneu-WREX is a lightweight exoskeleton that allows a wide range-of-motion of the arm in 3-D space and can apply relatively large forces (upwards of $40 \mathrm{~N}$ ) to the arm with a bandwidth of about $6 \mathrm{~Hz}$. Pneu-WREX also includes multiple redundant hardware and software safety features.

The adaptive controller that we designed to assist patients in moving is a Lyapunov based algorithm using the sliding surface developed in [32]. The full Lyapunov function candidate used for the Pneu-WREX orthosis contains terms necessary for both

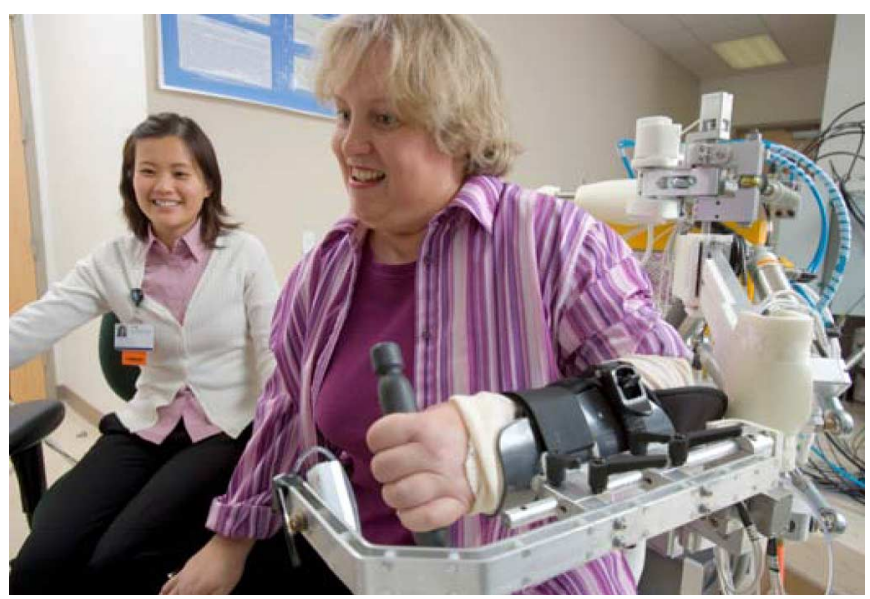

Fig. 1. Pneu-WREX: A four degree-of-freedom pneumatically actuated upper extremity orthosis for robot-aided movement training.

the orthosis dynamics and the force dynamics of the pneumatic actuators (see [29] for details). The following section briefly describes the model based adaptive controller and the adaptation to make it provide "assistance-as-needed."

\section{B. Assist-as-Needed Controller}

During robot-aided movement training the adaptive controller applies an $n \times 1$ vector of assistance forces $\mathbf{F}_{r}$ in spatial coordinates $\mathbf{x}$ at the location of the interface between the subject and the robot. $\mathbf{F}_{r}$ is determined using a model based adaptive control law, as in [32] and defined

$$
\begin{aligned}
\mathbf{F}_{r} & =\mathbf{Y} \hat{\mathbf{a}}-\mathbf{K}_{P}\left(\mathbf{x}-\mathbf{x}_{\mathbf{d}}\right)-\mathbf{K}_{D}\left(\dot{\mathbf{x}}-\dot{\mathbf{x}}_{\mathbf{d}}\right) \\
& =\mathbf{Y} \hat{\mathbf{a}}-\mathbf{K}_{P} \tilde{\mathbf{x}}-\mathbf{K}_{D} \dot{\tilde{\mathbf{x}}}
\end{aligned}
$$

where $\mathbf{x}$ and $\mathbf{x}_{d}$ are $n \times 1$ vectors of the actual and desired location of the hand, respectively, $\mathbf{K}_{P}$ and $\mathbf{K}_{D}$ are symmetric, constant, positive definite gain matrices, $\mathbf{Y}$ is a $n \times m$ matrix of known functions of $\mathbf{x}, \dot{\mathbf{x}}, \mathbf{x}_{d}, \dot{\mathbf{x}}_{d}$, and $\ddot{\mathbf{x}}_{d}$ and $\hat{\mathbf{a}}$ is an $m \times 1$ vector of estimates of the real system parameters $\mathbf{a}$. The desired trajectory of the hand $\mathbf{x}_{d}$ is generated from an interactive computer game and displayed as a cursor on a monitor, as shown in Fig. 2.

In (1), $-\mathbf{K}_{P} \tilde{\mathbf{X}}$ and $-\mathbf{K}_{D} \dot{\tilde{\mathbf{X}}}$ are proportional feedback terms and $\mathbf{Y a}$ is feedforward model of the dynamics of the robot orthosis and human arm combination including the output of the subject. The values of $\mathbf{K}_{P}$ and $\mathbf{K}_{D}$ used in experimental testing with Pneu-WREX are given in Fig. 2. These values were chosen by trial-and-error experimentally, such that the orthosis had a soft, slightly under-damped feel.

The regressor matrix $\mathbf{Y}$ is typically derived using classical dynamic modeling techniques. For robot-aided movement training, however, a more general modeling approach is needed because the arm dynamics and movement ability of a person who has suffered a stroke vary widely based on the location of the subject's hand in task space and from subject to subject. The goal, then, is to design the regressor matrix $\mathbf{Y}$ so that it fully spans the impairment space of subjects who have impairment due to stroke. To do this, we implemented a general 


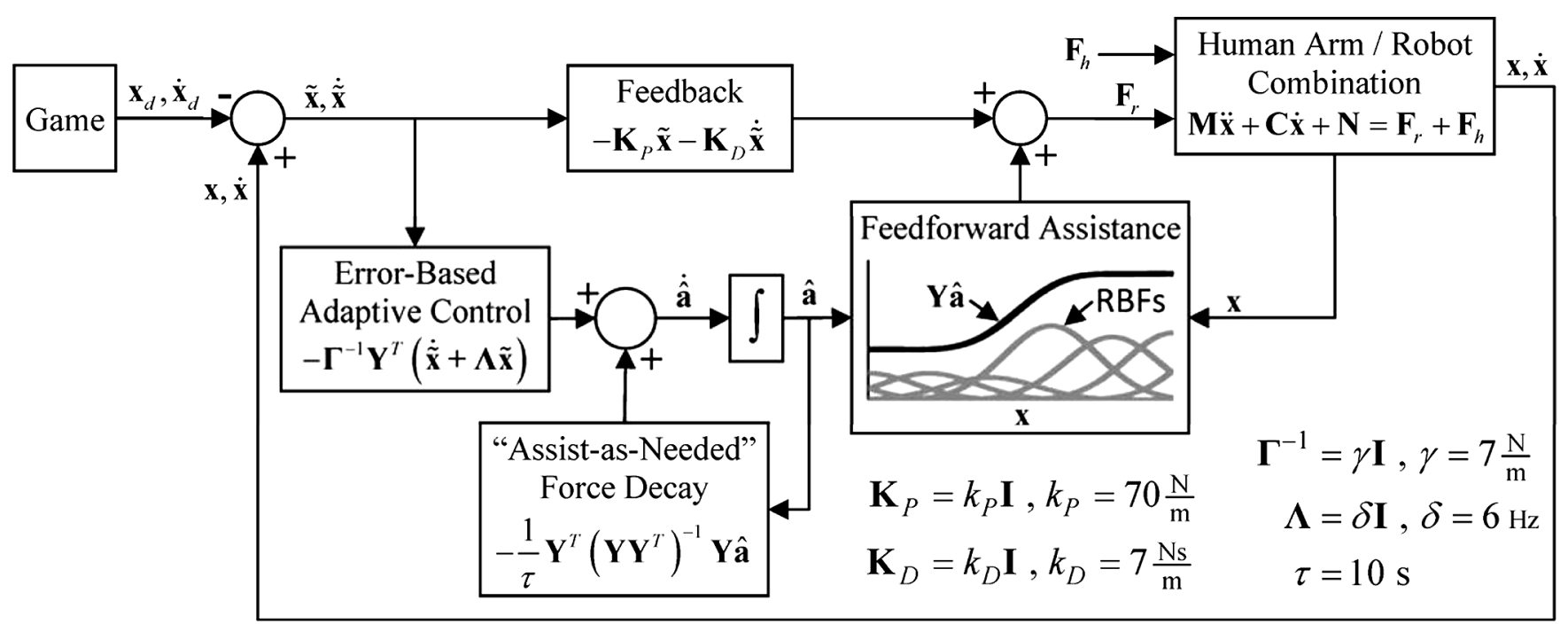

Fig. 2. Controller diagram. The "assist-as-needed" force decay term continuously reduces the feedforward assistance when errors are small. Feedforward assistance is a learned model of the subject's abilities and effort using radial basis functions. Gains of the controller are given in the figure. The effective integral, proportional, and derivative gains, taking into account the adaptive action of the controller, are derived in the Appendix.

representation of a patient's ability and tone using Gaussian radial basis functions so that

$$
\mathbf{Y}^{n \times m}=\left[\begin{array}{c:c:c:c}
\mathrm{g}^{T} & 0 & \cdots & 0 \\
\hdashline 0 & \mathbf{g}^{T} & \cdots & 0 \\
\hdashline \vdots & \vdots & \ddots & 0 \\
\hdashline 0 & 0 & 0 & \mathbf{g}^{T}
\end{array}\right]
$$

where $\mathrm{g}$ is a $p \times 1$ vector of radial basis functions with $m=n p$. With this selection for $\mathbf{Y}$, the parameter vector â becomes a vector of amplitudes of the basis functions that determines how they are blended to produce the feedforward estimate of force. The radial basis functions are distributed evenly in a spatial grid that spans the workspace of the orthosis. Each spatial grid point contributes to the assistive force output based on the location of the orthosis in task space. Details of the implementation for Pneu-WREX are given in the Appendix.

In order to limit computational complexity, the current implementation of Pneu-WREX does not include inertia components in the regressor matrix $\mathbf{Y}$. This also avoids potential instabilities from unmodeled dynamics (as present in all real systems) that can be triggered from imperfect velocity and acceleration signals. The effect of the omission of inertia components is minor because movement speeds for robot-aided movement training are relatively low. Future implementations could implement inertia components by adding additional dimensions to the Gaussian radial basis functions.

To allow the controller to adapt to the patient, the modified parameter estimates $\hat{\mathbf{a}}$ are updated according to

$$
\dot{\hat{\mathbf{a}}}=-\frac{1}{\tau} \mathbf{Y}^{T}\left(\mathbf{Y} \mathbf{Y}^{T}\right)^{-1} \mathbf{Y} \hat{\mathbf{a}}-\Gamma^{-1} \mathbf{Y}^{T}(\dot{\tilde{\mathbf{x}}}+\Lambda \tilde{x})
$$

where $\boldsymbol{\Lambda}$ and $\boldsymbol{\Gamma}$ are $n \times n$ symmetric, constant, positive definite, gain matrices and $\tau$ is the time constant for the decay of the force output. $\Gamma$ determines the overall error based adaptation rate and $\boldsymbol{\Lambda}$ specifies the ratio of position error adaptation to velocity error adaptation. For Pneu-WREX, $\boldsymbol{\Lambda}=\delta \mathbf{I}$ and $\boldsymbol{\Gamma}=\gamma \mathbf{I}$. Although in theory $\delta$ and $\gamma$ can be any positive value, in practice the presence of unmodeled dynamics limits the stable range of these parameters. For Pneu-WREX, the values for $\delta$ and $\gamma$ were chosen experimentally and are given in Fig. 2.

The first and second terms on the right side (3) create an adaptive controller with competing interests, with the first term attempting to reduce effort and the second term attempting to reduce error. The second term on the right side of (3), $-\Gamma^{-1} \mathbf{Y}^{T}(\dot{\tilde{\mathbf{x}}}+\Lambda \tilde{\mathbf{x}})$ is the standard adaptive control law, as in [32], and is required for the Lyapunov stability analysis presented in the Appendix. This second term adapts the parameters based on trajectory tracking error.

The first term on the right side of $-(1 / \tau) \mathbf{Y}^{T}\left(\mathbf{Y} \mathbf{Y}^{T}\right)^{-1} \mathbf{Y} \hat{\mathbf{a}}$ is the "assist-as-needed" modification to the standard adaptive control law. This novel term decays the force output with a time constant of $\tau$. The matrix $\mathbf{Y}^{T}\left(\mathbf{Y} \mathbf{Y}^{T}\right)^{-1} \mathbf{Y}$ limits the change in the parameter estimates a to those with the largest current influence on the output force. This keeps the parameter decay local with respect to the state of the regressor matrix $\mathbf{Y}$. For the implementation in Pneu-WREX, this means that the force decay affects the parameter estimates associated with radial basis functions that are spatially close to the position of the hand of the subject, with the parameter decay decreasing as the distance from the hand to the associated radial basis function increases. The derivation of this term is given in the Appendix.

A Lyapunov stability analysis (see the Appendix) of the controller defined by (1) and (3) shows stability of the system in the sense of uniform ultimate boundedness. This bound is a function of the time constant $\tau$ and the adaptation matrix $\Gamma$. In practice, the forgetting rate $1 / \tau$ weighs the balance between tracking error and robotic assistance and can be set based on experimental observation, as described below. 


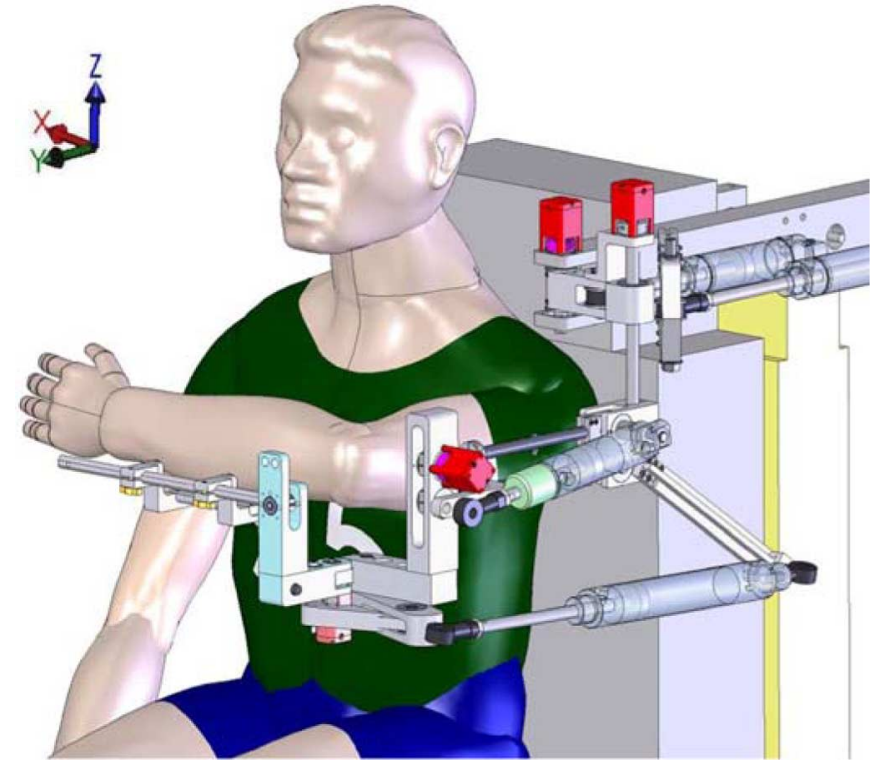

Fig. 3. Computer-aided design model and coordinate axes for Pneu-WREX. Origin of the coordinate system is located in the center of the subject's impaired side shoulder.

\section{Experimental Methods}

We tested the adaptive, assist-as-needed controller with 11 volunteer subjects who have upper extremity movement impairment due to chronic (six months post) stroke. All experiments were approved by the Institutional Review Board of the University of California, Irvine, and subjects provided informed consent.

Two separate experiments, named the spatial tracking experiment and the side-to-side experiment were used to evaluate the effectiveness of the controller. The primary goal of the spatial tracking experiment was to validate that the device had the ability to help subjects to complete desired movements across a wide workspace even when they were severely impaired. The goal of the side-to-side experiment was to rigorously test whether the assist-as-needed modification increased the contributions of the subjects to reaching movements.

During these experiments the patients were seated in a chair with their impaired arm attached to the orthosis with a forearm cuff. Straps were not used to restrain the trunk. We have found that subjects sometimes use such straps for leverage by leaning into them, and are better reminded to just use the arm to move by verbal cueing.

\section{Spatial Tracking Experiment Protocol}

In the spatial tracking experiment, the subjects controlled the location of a cursor on a computer screen by changing the location of their hand in task space. The subjects were instructed to track a target cursor on the computer screen as it moved back and forth from a central home position to seven spatially located targets. The target moved in the frontal plane of the subjects, with positive $x$ to the subject's right and positive $z$ in the upward vertical direction, as shown in Fig. 3. The origin of the workspace frame was the center of the subject's impaired side shoulder. The range of motion of the target in the $x$ direction was $55 \mathrm{~cm}$ and in the $z$ direction was $13 \mathrm{~cm}$. The movement of the hand away from the subject in the $y$ (forward-backward) direction was not controlled, so subjects moved to a naturally comfortable position in the $y$ direction. Note that the vertical force required from the robot to support the arm does not depend on elbow flexion angle, due to use of a four-bar mechanism at the shoulder [31]. The target cursor followed a minimum jerk trajectory in the $x-z$ plane with a peak velocity of $0.12 \mathrm{~m} / \mathrm{s}$ to and from each target. The seven targets were presented in a fixed order, starting with the bottom left target, followed by the bottom right target, then to the second lowest left target, and so on until the seventh target located directly above the home position. The tracking for all seven targets was repeated three times.

This experiment was repeated for two conditions. In the first condition (without assistance condition), the subjects attempted to track the target without assistance from the robot. During this condition, the robot operated in "backdrive" mode, with the desired output force set to zero. In this mode, the orthosis balanced its own weight, and the net force on the subjects, excluding the inertia effects of the orthosis, was approximately zero. Because this task was very difficult for these substantially impaired subjects, each subject was only asked to move to each target one time. For the second condition (with assistance condition), the robot assisted the subjects in tracking the target using the adaptive, assist-as-needed controller, as described in the previous sections. For this condition, the subjects reached to all seven targets three times. This condition was evaluated with a forgetting rate $1 / \tau$ equal to 0.1 (or $\tau=10 \mathrm{~s}$ ), as well as without a forgetting rate $(1 / \tau=0$ or $\tau=\infty)$. The value of $1 / \tau=0.1$ was chosen based on trial-and-error in pilot experiments so that the orthosis would successfully reach the targets (which were spherical with a diameter of $2.5 \mathrm{~cm}$ ) even if the subject was passive in the orthosis. Specifically, several pilot subjects were instructed to remain passive, and $1 / \tau$ was slowly increased until the controller could no longer move the arm into the target. The value for $1 / \tau$ that still allowed the orthosis to move into the target was used for subsequent experiments.

\section{E. Side-to-Side Experiment Protocol}

In the second experiment (side-to-side experiment), the subjects were instructed to track the target cursor back and forth between two targets. As in the first experiment, the subjects controlled a cursor on the computer screen by changing the location of their hand in task space while attempting to track the target cursor as it moved from side to side in the $x$ direction. The two targets were spaced $30 \mathrm{~cm}$ apart at chest level in the $x-z$ plane and had a size of $2.5 \mathrm{~cm}$. The target cursor followed a minimum jerk trajectory between the two targets with a peak velocity of $0.12 \mathrm{~m} / \mathrm{s}$. As in the previous experiment, the reach of the hand away from the subject in the $y$ direction was not controlled, so subjects' moved to a naturally comfortable position in the $y$ direction. The subjects tracked the target cursor back and forth between the two targets a total of 20 times. For this experiment, the adaptive, assist-as-needed controller was always used to help the subjects move back and forth between the two targets, but we measured performance of the controller without forgetting, and with the forgetting rate set to 0.1 . 


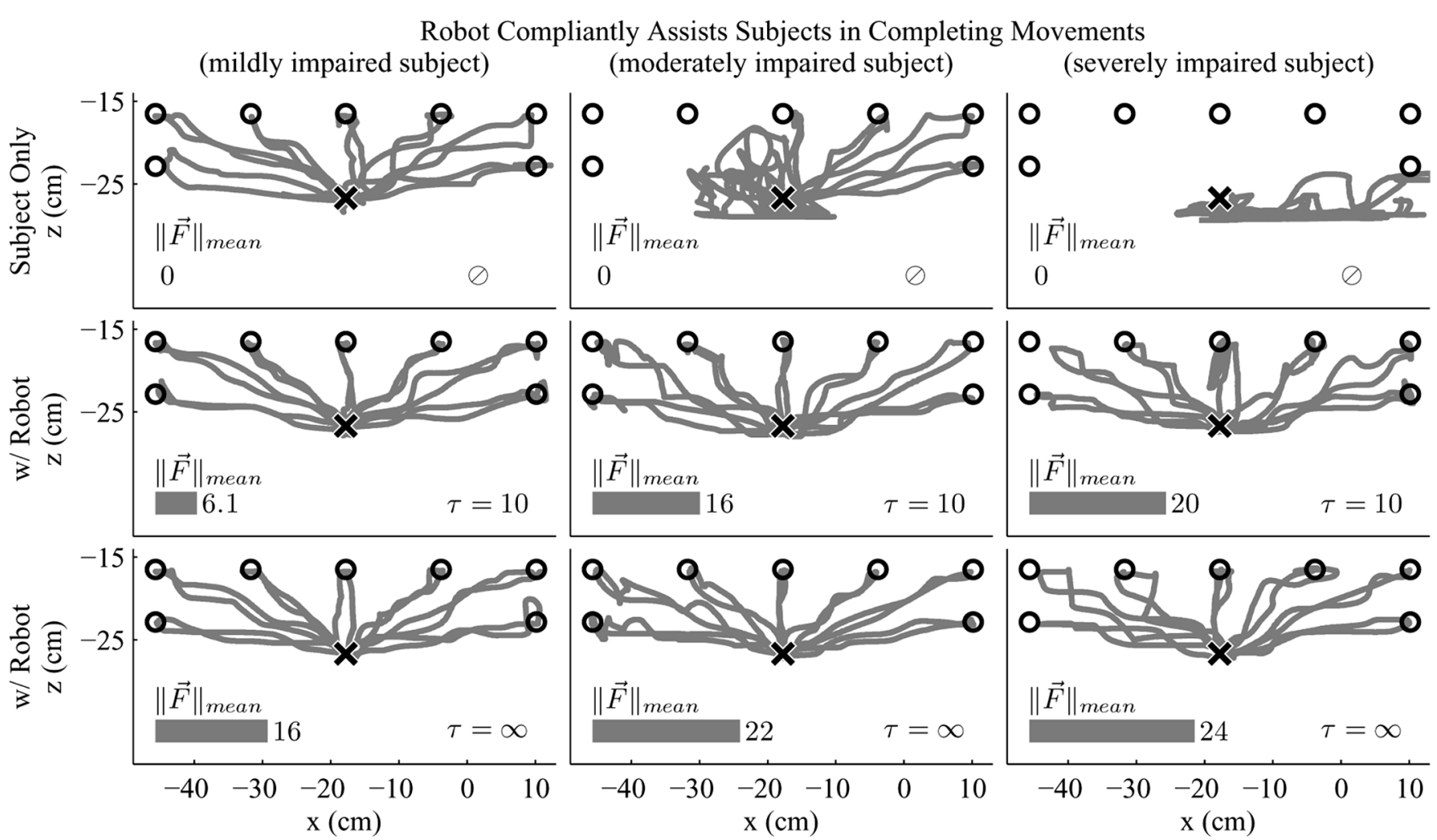

Fig. 4. Subject tracking performance and controller output for the spatial tracking experiment for mildly (Fugl-Meyer arm score $=53$, left column), moderately $(\mathrm{FM}=30$, middle column), and severely $(\mathrm{FM}=16$, right column) impaired subjects (using the Fugl-Meyer arm assessment score, $0=$ total impairment, $66=$ no impairment). Top row shows results for the without assistance condition, where the orthosis was in backdrive mode (for all three subjects combined the mean output force magnitude from the device in this mode was $0.46 \mathrm{~N}$ with a standard deviation of $0.46 \mathrm{~N}$ ). Bottom two rows show the results for the with assistance condition, with forgetting ( $\tau=10$, middle row), and without forgetting $(\tau=\infty$, bottom row). For the without assistance condition, the trajectories are plotted from a single repetition of all seven targets (without assistance, the task was too difficult for the subjects to repeat). For the with assistance condition, the results are from the third repetition of all seven targets. Targets are shown as circles and the central home position is shown as a cross. Mean of the controller output force magnitude during the shown movements is represented by the bar graph in the bottom left corner of each plot. This force is a rough indicator of the assistance provided to the subjects during the movements.

This second experiment was repeated for two conditions. In each condition, the forgetting rate $1 / \tau$ was set to 0 and 0.1 on two different trials. For the first condition (always active condition), the subjects were instructed to track the target cursor back and forth between the two targets 20 times. The purpose of this condition was to see if the adaptive, assist-as-needed controller could learn the forces necessary to assist the subjects in achieving the desired back and forth movements while allowing the subjects to remain as actively involved in the movements as possible.

In the second condition (relaxed to active condition), the subjects were instructed to relax their arms for the first five back and forth motions of the target cursor, letting the robot complete the movements for them. During these first five movements, the adaptive controller learned a model of the forces necessary to lift the subject's ("passive") arm and move it back and forth between the targets. After the completion of the fifth back and forth motion, the subjects were instructed to actively track the target cursor. The goal of this condition was to determine if the controller would reduce its force output to allow the subject to complete as much of the task as possible on his or her own.

\section{F. Data Collection and Analysis}

Sensor data from the robot for both the spatial tracking and side-to-side experiment were collected at $1 \mathrm{kHz}$. In both exper- iments, position and velocity in the horizontal and vertical ( $x$ and $z$ directions) were recorded. Additionally, the spatial force applied to the subject's arm by the robot in the horizontal and $\operatorname{vertical}(1 / \tau$ and $\tau=10$ directions) were also recorded, as measured by pressure transducers in the orthosis actuators.

\section{RESULTS}

We designed a controller for a robotic therapy device that learns a model of the subject's movement ability in real time using a radial-basis function representation, and then uses this model to provide compliant assistance for desired movements. We included a novel forgetting term in the controller so that it would optimize its assistance levels to the subjects' ability. Two experiments were performed to evaluate the effectiveness of this adaptive, assist-as-needed controller. The primary goal of the first experiment was to determine if the compliant, adaptive controller could assist subjects in completing desired movements across a wide range of the workspace, and the primary goal of the second experiment was to determine whether the addition of the forgetting term improved the ability of the controller to provide assistance-as-needed.

\section{A. Spatial Tracking Experiment}

Example data from the spatial tracking experiment are shown in Fig. 4. The controller successfully assisted subjects with 

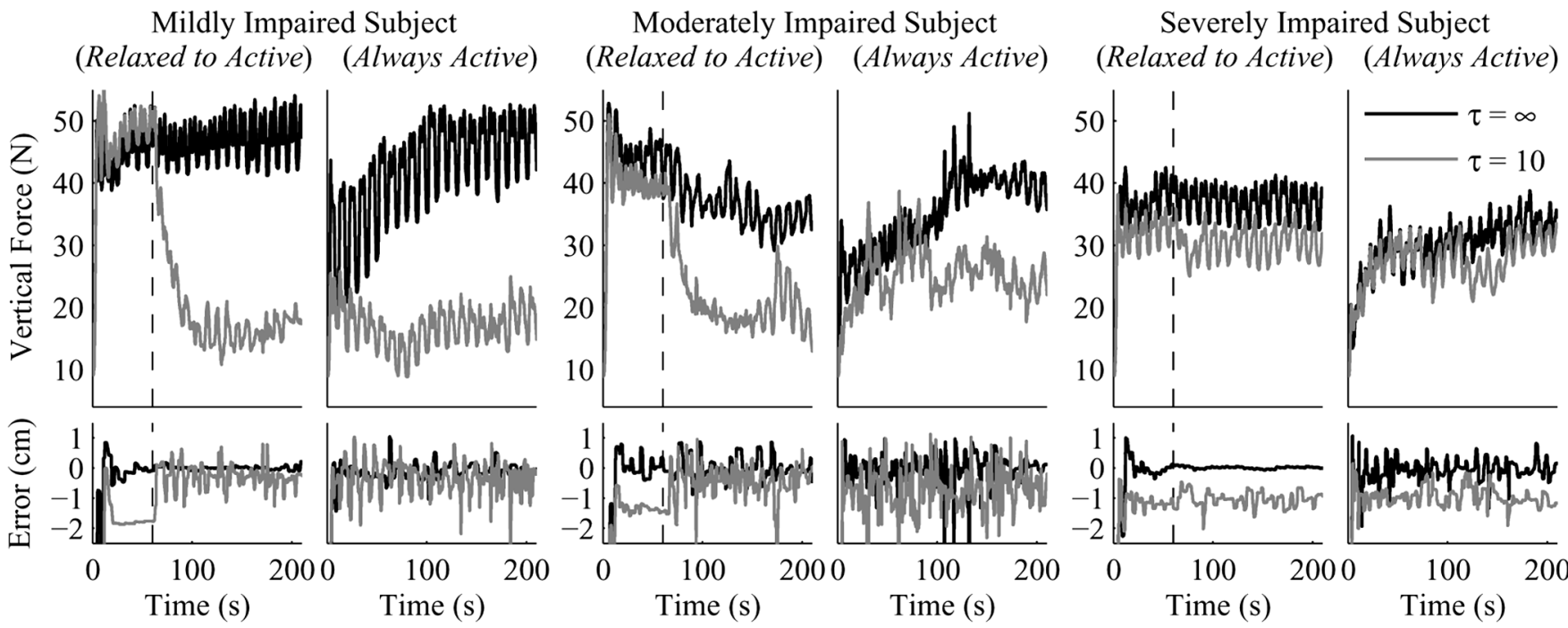

Fig. 5. Vertical assistance force and vertical tracking error during the side-to-side experiment for subjects with mild $(\mathrm{FM}=53$, left main column), moderate ( $\mathrm{FM}=30$, center main column), and severe ( $\mathrm{FM}=16$, right main column) impairment. Each main column shows results from a single subject for both relaxed to active (left subcolumn) and always active (right subcolumn) conditions. Dotted line marks the end of the fifth side to side movement for the relaxed to active condition.

various impairment levels in performing spatial movements to seven widely-spaced targets (Fig. 4 bottom two rows). Indeed, the controller successfully allowed all 11 subjects to reach all of the spatial targets. While doing so, the adaptive, assist-as-needed controller remained compliant, allowing variations in the actual movement trajectories regardless of assistance level (see examples in Fig. 4). Including the forgetting term in the controller also decreased the force output from the robotic orthosis during these movements, compared to when forgetting was excluded (compare bars in middle row to bottom row in Fig. 4), a phenomenon that we quantified more precisely in the next experiment.

\section{B. Side-to-Side Experiment}

We next asked subjects to reach repeatedly between only two targets in order to more precisely quantify the effect of forgetting and the real-time development of the robotic assistance force. The forces required to move the arm in the left-right horizontal $(x)$ direction were relatively low $(<2 N)$ and a significant difference was not seen in the effects of forgetting in that direction. However, in the vertical direction, where the weight of the subject's arm (typically $\sim 40 \mathrm{~N}$ ) needed to be overcome, the effects of forgetting were evident. The vertical assistance force and vertical tracking error from the side-to-side experiment for a mildly impaired subject, a moderately impaired subject, and a severely impaired subject are shown in Fig. 5. For the relaxed to active condition the adaptive, assist-as-needed controller quickly learned the vertical force required to support the subject's relaxed arm during the side-to-side movements, both when the controller included forgetting and when it did not. The vertical tracking error was slightly larger $(<2 \mathrm{~cm})$ when the forgetting term was included (consistent with in the Appendix, which predicts tracking error to be proportional to the weight of the subject's arm.) Once the subject began to actively participate in the movements, the force output of the controller depended strongly on whether the forgetting term was present. With a forgetting term included, the controller reduced its force output while keeping the tracking errors small, producing a larger effort level from the subject. The amount of force reduction from the controller once the subject began to try to move depended on the ability of the subject, which can be seen by comparing the relaxed to active plots of Fig. 5 for each subject and observing that the force output from the controller decreased with the decreasing level of the subject's movement impairment. In contrast, when forgetting was omitted, the force output from the controller remained high regardless of the ability level of the subject, indicating that the subjects relied on the robotic orthosis for most of the vertical support needed during the side-to-side movements.

A similar pattern of assistive force was present in the always active condition (Fig. 5, right side subcolumns). In this condition, the subjects actively participated in all of the side-to-side movements from the beginning. When a forgetting term was included, the robot assistance force increased from its initialized zero value, by an amount depending on the impairment severity of the subject, to the level necessary to keep tracking errors small. Conversely, when the forgetting term was not included, the output force slowly increased to a level equivalent to that necessary for when the subjects relaxed their arms during the relaxed to active condition. Thus, without forgetting, the robot essentially "took over" the task of arm support during the side-to-side movements.

For both relaxed to active and always active conditions, the final vertical assistance force from the controller converged to a steady state value that depended on both the forgetting rate $1 / \tau$ and the impairment severity of the subject. To quantify this steady state value more precisely, Fig. 6 shows the mean vertical assistance and mean error for the last two side-to-side movements of the always active Condition from all 11 subjects plotted versus arm impairment score (quantified using the Fugl-Meyer arm assessment). Incorporating a forgetting factor into the adaptive controller caused it to provide a steady state assistance force that varied linearly with arm impairment (circles 


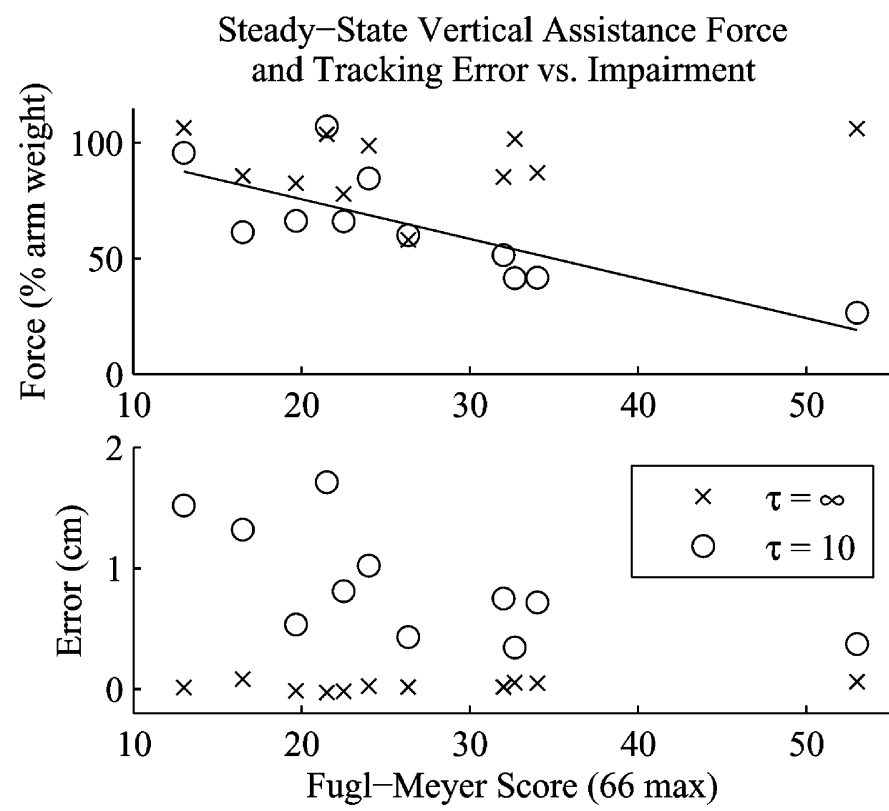

Fig. 6. Mean vertical robotic assistance force and mean tracking error during side-to-side reaching experiment. Average force (as a percentage of arm weight) and average error for the last two movements of the always active condition for the side-to-side experiment are plotted for each subject versus arm impairment (using the Fugl-Meyer arm assessment score, $0=$ total impairment, $66=$ no impairment.) A regression line of the assistance force is plotted for the case of $\tau=10$.

in the top plot of Fig. $6, R^{2}=0.6, p<0.006$ ). Thus, the controller required less impaired subjects to do more of the work of lifting their arms themselves, and thus more fully engaged the subjects in the movement task. Note that when forgetting was included, the tracking error was larger than without forgetting, but still relatively small (under $2 \mathrm{~cm}$.)

When forgetting was not included, however, the controller provided a significantly larger amount of assistance (paired $t$-test, $p<0.008$ ). The assistance level was nearly $100 \%$ of the weight of the arm, regardless of the subject's impairment level. Thus, the standard adaptive controller (i.e., the controller without forgetting) essentially "took-over" the task for the subject, reducing the participation level of the subject.

\section{DISCUSSION AND CONCLUSION}

We developed a controller that exhibits the characteristics that we hypothesize are important for maximizing the therapeutic benefit of robot-assisted movement training. The controller is theoretically stable, as shown using a Lyapunov-based analysis. It is capable of compliantly assisting patients with a force tailored to their impairment level, as shown experimentally. The foundation for this control framework is an adaptive controller that learns in real time a model of the patient's ability and effort. The controller uses tracking error to drive model formation, and therefore learns quickly on a patient-specific, session-specific, manner with only simple, kinematic information about patient performance. The developed controller adapts in real time, removing the need to change parameters from patient to patient and/or from therapy session to therapy session, or when the abilities of a patient change. The model based component of the controller continuously adapts, assisting more when errors are large and letting the subject do more when errors are small. The controller compliance is independent of the model based assistance, and can therefore be kept high. Because of this high compliance, patients subjectively reported that they "felt in control" during movement training.

Use of adaptive control techniques for robotic therapy has been previously suggested [6], [33], but to our knowledge a controller that forms a real-time model of the patient's force producing capability has not previously been achieved. Such a controller likely mimics what a human therapist does as he or she assists a patient. That is, it is well established that humans learn to move in novel but predictable dynamic environments by adaptively forming an internal model of the environment, rather than relying on stiff position control (for review, see [26]). To an assisting therapist, the patient's limbs are a novel dynamic environment. Therefore, therapists almost certainly adaptively form internal models of the patients' limbs to assist them in achieving desired movements, and the controller proposed here mimics that process.

An important finding in the present study within the context of this model-based paradigm is that the human motor control system takes advantage of a standard, model-based adaptive controller to reduce its own effort, letting the controller "take over" most of the physical effort required to complete the movement. This observation of human "slacking" is consistent with a large body of research that suggests that the human motor system optimizes effort [36]. Recent results from our laboratory indicate how the unimpaired motor system achieves this optimization, at a computational level, for the task of adapting to a novel dynamic environment [37]. The human motor system slacks (i.e., minimizes effort) by incorporating a forgetting factor similar to the one we incorporated into the adaptive controller. The finding of human slacking in the present study is also consistent with the recent finding that spinal cord injured patients expended $60 \%$ less energy during walking on a treadmill when assisted by a stiff, nonadaptive robotic gait training machine, when compared to a compliant, adaptive group of human therapists [22].

If one accepts the premise that patient participation and effort are important for provoking neural plasticity during rehabilitation therapy, a standard, model-based adaptive controller would therefore be suboptimal for movement training, even though it successfully allows patients to complete desired movements. As shown here, a solution to this problem is to include a forgetting rate that decays the forces applied to the patient's extremity when errors are small. The inclusion of the forgetting rate makes the robot minimize force in a way analogous to the way that humans apparently do (including rehabilitation therapists, of course) [9]. The resulting controller forms a model of the patient's movement ability while encouraging patient effort by providing a form of "assistance-as-needed."

Comparing the performance of the presented controller with other "assist-as-needed" control approaches highlights its advantages. For example, in comparison with a standard impedance controller without a feedforward term, the present controller achieves much better tracking across a 3-D workspace than normally would be possible with a low level of impedance. For example, the adaptive controller used here 
had an effective proportional position gain of $109 \mathrm{~N} / \mathrm{m}$ (see the Appendix for effective gain calculation). Given that it took about $40 \mathrm{~N}$ for the robot to lift a patient's relaxed arm to a target (Fig. 5), then an impedance controller without a feedforward term would have a tracking error of $0.37 \mathrm{~m}$ in the vertical direction, clearly unacceptable. Tracking error could be reduced by increasing the stiffness of an impedance controller considerably, but then the robot would rigidly drive patient movements, losing the hypothesized benefits of compliant assistance for neural plasticity. Tracking error could also be reduced by adding a feedforward term to an impedance controller to help compensate for the weight of the arm (such as by adding a constant offset equal to the weight of the arm [15], or a fixed model of the weight of the arm [16], [38]). However, this approach requires manually adjusting the feedforward term for each patient. More importantly, this approach fails to take into account that patients can contribute to lifting their arms themselves, increasingly so as they recover. The controller presented here provides a means to both automatically create the feedforward term across a wide workspace in real-time, and then to automatically reduce it as the patient recovers.

Previous attempts to implement assistance-as-needed for robotic therapy have also taken the approach of adapting control parameters such as the stiffness of the robot [23], [41], the shape [42], or timing [20], [23], [43] of the desired trajectory, or parameters of a viscous force field that assists in movement [24] from trial-to-trial based on ongoing measurement of performance error. The controller presented here is different in that it adapts the amount of force assistance provided by the robot by adjusting a model of the patient's weakness from movement to moment based on tracking error. Again, this allows the robot to remain compliant as explained above, which is an advantage over these previous performance-based adaptive approaches. We had previously developed a simple version of such a model-based, assist-as-needed controller for the task of adapting to a robotic force field during walking, providing conceptual guidance for the present study [37]. Here, however, we showed for the first time how to achieve model-based, "assistance-as-needed" for arbitrary spatial movements, including ones that are common to upper extremity rehabilitation paradigms, and confirmed the presence of human slacking during model-based assistance of such rehabilitation-type movements. We are working to develop models that explain the neurocomputational dynamics at play [30].

An adaptive controller with some of the same features as the one proposed here was recently proposed by Mihelj et al. [39]. This controller automatically adjusts a vertical support force based on tracking error, and includes a forgetting factor so that the support force decreases when vertical tracking error is small. The present controller is different, however, in that it forms a model of the support forces needed in all directions, as a function of the arm state. In addition, the model is updated in a way in which stability can be proven, as shown here. Further, we show here experimentally that the forgetting factor is a necessary addition, otherwise the patient slacks.

The adaptive controller presented here adapts quickly to the patient's abilities-within the course of an ongoing movement. In contrast, the changes in movement ability associated with recovery take place over a much longer time constant-across days. Thus, the quasi real-time adaptation of the present controller is likely quicker than is necessary to adjust for ongoing motor recovery. However, such fast adaptation is not a disadvantage with respect to accommodating motor recovery, and has the advantage that it also enables the device to compensate for changes in motor output that occur on a faster time scale, such as those due to moment-to-moment changes in muscle tone or fatigue.

We did not test the therapeutic efficacy of the proposed, model-based, assist-as-needed controller here, and the ultimate validation of the controller will be such testing. We note that the results presented here lay the groundwork for a rigorous test of the role of patient effort in rehabilitation efficacy. Specifically, the control framework developed here allows patients to complete desired movements with ostensibly equivalent kinematics, but while contributing different levels of force to the task, depending on how the forgetting rate is set. By studying two groups of patients that train with two different forgetting rates (and thus two different levels of effort), one should, therefore, be able to test the role of effort in recovery, in isolation from the confounding changes in movement repetition or kinematics that are usually associated with changes in effort. This possibility highlights the role that robotic therapy devices will likely increasingly play in progressing rehabilitation science. The ability to program robotic therapy devices to algorithmically isolate and manipulate key aspects of motor training will allow rigorous testing of whether, and how much, those aspects influence motor plasticity.

\section{APPENDIX}

\section{Mathematical Details of Adaptive Control Algorithm}

\section{A. Combined Orthosis and Arm Dynamics}

The rigid body dynamics of a robotic orthosis when connected to a human subject can be defined in task coordinates as

$$
\mathbf{M}(\mathbf{x}) \ddot{\mathbf{x}}+\mathbf{C}(\mathbf{x}, \dot{\mathbf{x}}) \dot{\mathbf{x}}+\mathbf{N}(\mathbf{x}, \dot{\mathbf{x}})=\mathbf{F}_{r}+\mathbf{F}_{h}
$$

where $\mathrm{x}$ is a $n \times 1$ vector of task space coordinates specifying the position and orientation of the hand (for Pneu-WREX $n=4$ and $\left.\mathbf{x}=\left[\begin{array}{llll}x & y & z & \theta_{z}\end{array}\right]^{T}\right), \mathbf{F}_{r}$ is an $n \times 1$ vector of forces applied by the robot actuators which is mapped by the Jacobian to the interface location, $\mathbf{F}_{h}$ is an $n \times 1$ vector of forces the patient can contribute to the desired movement, $\mathbf{M}$ is the $n \times n$ generalized inertia matrix, $\mathbf{C}$ is the $n \times n$ Coriolis matrix, and $\mathbf{N}$ is an $n \times 1$ vector of external forces acting on the robotic orthosis and human arm combination, including gravity and static forces arising from tone, and viscous and friction forces.

\section{B. Adaptive Controller for Movement Training}

The adaptive controller design uses the sliding surface $\mathbf{s}$ and reference trajectory $\mathbf{w}$ as in [32]. $\mathbf{s}$ and $\mathbf{w}$ are defined as

$$
\begin{aligned}
\mathbf{s} & =\dot{\tilde{\mathbf{x}}}+\Lambda \tilde{\mathbf{x}}=\left(\dot{\mathbf{x}}-\dot{\mathbf{x}}_{d}\right)+\boldsymbol{\Lambda}\left(\mathbf{x}-\mathbf{x}_{\mathbf{d}}\right) \\
\mathbf{w} & =\dot{\mathbf{x}}_{d}-\Lambda \tilde{\mathbf{x}}=\dot{\mathbf{x}}_{d}-\Lambda\left(\mathbf{x}-\mathbf{x}_{\mathbf{d}}\right)
\end{aligned}
$$


where $\mathbf{x}$ and $\mathbf{x}_{d}$ are $n \times 1$ vectors of the actual and desired location of the hand, respectively, and $\Lambda$ is an $n \times n$ symmetric, constant, positive definite, gain matrix. For actuators with negligible dynamics, such as electric motors, the desired robot force $\mathbf{F}_{r}^{d}$ equals the actual robot force $\mathbf{F}_{r}$. Therefore, the control law for this method is

$$
\mathbf{F}_{r}=\mathbf{F}_{r}^{d}=\mathbf{Y}(\mathbf{x}, \dot{\mathbf{x}}, \mathbf{w}, \dot{\mathbf{w}}) \hat{\mathbf{b}}-\mathbf{K}_{P} \tilde{\mathbf{x}}-\mathbf{K}_{D} \dot{\tilde{\mathbf{x}}}
$$

where $\mathbf{K}_{P}$ and $\mathbf{K}_{D}$ are symmetric, constant, positive definite gain matrices and $\mathbf{Y} \hat{\mathbf{a}}$ is a model of the system dynamics and human force output and is defined as

$$
\mathbf{Y} \hat{\mathbf{a}}=\hat{\mathbf{M}} \dot{\mathbf{w}}+\hat{\mathbf{C w}}+\hat{\mathbf{N}}-\hat{\mathbf{F}}_{h}
$$

where $\hat{\mathbf{M}}, \hat{\mathbf{C}}$, and $\hat{\mathbf{N}}$ are estimates of the dynamics of the robotic orthosis and human arm combination, $\mathbf{Y}$ is a $n \times m$ matrix of known functions of $\mathbf{x}, \dot{\mathbf{x}}, \mathbf{w}$, and $\dot{\mathbf{w}}$, and $\hat{\mathbf{a}}$ is an $m \times 1$ vector of estimates of the real system parameters, a. In effect, Yâ is feedforward model of the dynamics of the robot orthosis and human arm combination including the ability and effort of the subject.

For the current implementation of Pneu-WREX the regressor matrix Yâ is comprised of spatially dependent Gaussian radial basis functions, defined as

$$
g_{n}=\exp \left(-\frac{\left|\mathbf{x}-\boldsymbol{\mu}_{n}\right|^{2}}{2 \sigma^{2}}\right)
$$

where $g_{n}$ is the $n$th radial basis function, $\mathbf{x}$ is the current location of the patient's hand, $\boldsymbol{\mu}_{n}$ is the location of the $n$th radial basis function, and $\sigma$ is effectively a scalar smoothing constant that determines the width of the basis function. For Pneu-WREX, we have implemented a three-dimensional grid of radial basis functions, with eight grid divisions left to right $(-x$ to $+x)$, five grid divisions in and out $(-y$ to $+y)$, and three grid divisions down to up $(-z$ to $+z)$ across the workspace of the robot, and with $\sigma=7.62 \mathrm{~cm}$. The grid divisions are evenly spaced at $10 \mathrm{~cm}$ apart. The value of $\sigma$ changes the width of each radial basis function so it must be determined in conjunction with the grid spacing, so that there is sufficient overlap in the radial basis functions to achieve good function approximation. The grid spacing was chosen to be small enough to get reasonable spatial variance in the function approximation but without adding excessive computational expensive for real-time control. The vector of all of the Gaussian radial basis functions is defined

$$
\mathbf{g}=\left[\begin{array}{llll}
g_{1} & g_{2} & \ldots & g_{120}
\end{array}\right]^{T} .
$$

We combine this vector of Gaussian radial basis functions to define the regressor matrix $\mathbf{Y}$ as

$$
\mathbf{Y}^{3 \times 360}=\left[\begin{array}{c:c:c}
\mathbf{g}^{T} & 0 & 0 \\
\hdashline 0 & \mathbf{g}^{T} & 0 \\
\hdashline 0 & 0 & \mathbf{g}^{T}
\end{array}\right]
$$

The parameter estimate vector $\hat{\mathbf{a}}$ is, therefore, a $360 \times 1$ vector, with the parameters representing the amount of force the subject is unable to provide to hold their arm at a particular location in space. Including more parameters (e.g., more basis functions) is possible and would allow the model to represent more complicated impairment, but would also increase the computational expense. The standard parameter estimate update law for this method is

$$
\dot{\hat{a}}=-\Gamma^{-1} \mathbf{Y}^{T} \mathbf{s}
$$

where $\boldsymbol{\Gamma}$ is a symmetric, positive definite gain matrix. When all of the inertial and gravitational terms of (7) are included, and the force output from the human subject remains time independent, the controller defined by (6) and (11) is globally asymptotically stable, following an analysis similar to [44]. For the implementation with Pneu-WREX used here, however, the inertial components of the dynamic model were omitted for simplicity, and because the movements of interest were relatively slow. In addition, the output force from the human subject is most certainly time dependent. Thus, the resulting system is not globally asymptotically stable. However, it is possible to show that the system does exhibit uniform ultimate boundedness, with the tracking errors limited by the bounds of the system dynamics and by the bounds of the force output from the human subject. We have found experimentally that a controller that uses (11) without inertial components will converge to small steady state tracking errors.

\section{Assist-as-Needed Controller Modification}

We modified the parameter update law (11) in order to reduce the forces applied to the subject when errors are small. This modification decays the force applied by the robotic orthosis when the subject is able to complete movements without assistance. In order to achieve this decay, we specify that the partial derivative of the robot assistance force with respect to time should, when error is zero, behave according to

$$
\frac{\partial}{\partial t}(\mathbf{Y} \hat{a})=\mathbf{Y} \dot{\hat{\mathbf{a}}}=-\frac{1}{\tau} \mathbf{Y} \hat{\mathbf{a}}
$$

where Y $\mathbf{a}$ is the model based feedforward force applied to the subject's arm by the adaptive controller, and $1 / \tau$ is the forgetting rate of the robot $(\tau=$ time constant). Using the partial derivative of $\mathbf{Y} \hat{a}$ in (12) limits the change in $\mathbf{a}$ so that it is not a function of changes in the regressor matrix $\mathbf{Y}$, which is dependent on the state (position, velocity, and acceleration) and desired state of the end-effector. This allows information learned from previous motions to remain in the parameter vector â so that when the patient returns to similar motions later in time, the assistance learned from previous trials is still available.

In general, $\mathbf{Y}$ is an $n \times m$ matrix with $m>n$ and rank $n$. Thus, there are an infinite number of solutions for $\dot{\hat{a}}$ that satisfy (12). By seeking the minimum norm solution for $\dot{\hat{a}}$, the parameters that change are those that have the most influence in the force output at a given location. At the same time, parameters learned during other different motions remain unchanged. This allows the controller to learn a model of the neuromuscular weakness and patient effort as a general function of desired 
position, velocity, and acceleration of the hand in task space. The minimum norm solution for $\dot{\hat{a}}$ is found by solving the constrained minimization problem

$$
\min _{\dot{\hat{\mathbf{a}}}}:\left\{f=\frac{1}{2} \dot{\hat{\mathbf{a}}}^{T} \dot{\hat{\mathbf{a}}}: g=-\mathbf{Y} \dot{\hat{\mathbf{a}}}-\frac{1}{\tau} \mathbf{Y} \hat{\mathbf{a}}=\mathbf{0}\right\} .
$$

The minimizing solution to (13) is

$$
\dot{\hat{\mathbf{a}}}=-\frac{1}{\tau} \mathbf{Y}^{T}\left(\mathbf{Y} \mathbf{Y}^{T}\right)^{-1} \mathbf{Y} \hat{\mathbf{a}} .
$$

This term is added to the right side of (11) to create the modified parameter update law

$$
\dot{\hat{\mathbf{a}}}=-\frac{1}{\tau} \mathbf{Y}^{T}\left(\mathbf{Y} \mathbf{Y}^{T}\right)^{-1} \mathbf{Y} \hat{\mathbf{a}}-\Gamma^{-1} \mathbf{Y}^{T} \mathbf{s} .
$$

A Lyapunov stability analysis of the system with the modified parameter estimate update law is given in Appendix E.

\section{Effective Controller Gains}

The effective gains of the assist-as-needed adaptive controller can be determined by first taking the derivative of (6) to get

$$
\dot{\mathbf{F}}_{r}=\dot{\mathbf{Y}} \hat{\mathbf{a}}+\mathbf{Y} \dot{\hat{\mathbf{a}}}-\mathbf{K}_{P} \dot{\tilde{\mathbf{x}}}-\mathbf{K}_{D} \ddot{\tilde{\mathbf{x}}}
$$

Next, we substitute (3) into (16), which produces

$$
\dot{\mathbf{F}}_{r}=\dot{\mathbf{Y}} \hat{\mathbf{a}}-\frac{1}{\tau} \mathbf{Y} \hat{\mathbf{a}}-\Gamma^{-1} \mathbf{Y} \mathbf{Y}^{T}(\dot{\tilde{\mathbf{x}}}+\boldsymbol{\Lambda} \tilde{\mathbf{x}})-\mathbf{K}_{P} \dot{\tilde{\mathbf{x}}}-\mathbf{K}_{D} \ddot{\tilde{\mathbf{x}}} .
$$

We can now replace Yâ in (17) using (6) and rearrange terms so that we have

$$
\begin{aligned}
& \dot{\mathbf{F}}_{r}=\dot{\mathbf{Y}} \hat{\mathbf{a}}-\frac{1}{\tau} \mathbf{F}_{r}-\underbrace{\left(\frac{1}{\tau} \mathbf{K}_{P}+\boldsymbol{\Gamma}^{-1} \mathbf{Y} \mathbf{Y}^{T} \boldsymbol{\Lambda}\right)_{\mathbf{G}_{I}}} \tilde{\mathbf{\mathbf { x }}} \\
&-\underbrace{\left(\frac{1}{\tau} \mathbf{K}_{D}+\boldsymbol{\Gamma}^{-1} \mathbf{Y} \mathbf{Y}^{T}+\mathbf{K}_{P}\right)_{\mathbf{G}_{P}}} \dot{\tilde{\mathbf{x}}}-\underbrace{\mathbf{K}_{D}}_{\mathbf{G}_{D}} \ddot{\tilde{\mathbf{x}}}
\end{aligned}
$$

where $\mathbf{G}_{I}, \mathbf{G}_{P}$, and $\mathbf{G}_{D}$ are the effective integral, proportional, and derivative gains of the system. For Pneu-WREX YYY ${ }^{T}$ is a unitless $3 \times 3$ gain matrix defined by the spacing between radial basis functions and the smoothing parameter, $\sigma$. Because the grid points are evenly spaced, $\mathbf{Y} \mathbf{Y}^{T}=v I$ (for Pneu-WREX, $v=5.45$ ). The even grid spacing has the same effect on the effective gains, so that

$$
\begin{aligned}
\mathbf{G}_{I} & =g_{I} \mathbf{I}, \quad g_{I}=\frac{k_{P}}{\tau}+\gamma v \delta \\
g_{I} & =\left(\frac{7}{10}+7(5.45) \frac{1}{6}\right) \frac{\mathrm{N}}{\mathrm{ms}}=13.4 \frac{\mathrm{N}}{\mathrm{ms}} \\
\mathbf{G}_{P} & =g_{P} \mathbf{I}, \quad g_{P}=\frac{k_{D}}{\tau}+\gamma v+k_{P} \\
g_{p} & =\left(\frac{7}{10}+7(5.45)+70\right) \frac{\mathrm{N}}{\mathrm{m}}=109 \frac{\mathrm{N}}{\mathrm{m}} \\
\mathbf{G}_{D} & =g_{D} \mathbf{I}, \quad g_{D}=k_{D}=7 \frac{\mathrm{Ns}}{\mathrm{m}} .
\end{aligned}
$$

Of the other two terms in (18), only $\dot{Y} \hat{a}$ is a function of state (in this case, position). This term reflects how the learned assistance model changes the robot output force. Assuming this term is small, the effective gain values given (19) most accurately describe the impedance of the controller as experienced by the subject.

\section{E. Lyapunov Stability Analysis}

Stability is an important consideration for a machine that physically interacts with a human. This section details the Lyapunov stability analysis for the adaptive, assist-as-needed controller. The analysis builds on the adaptive algorithms developed by Slotine and by Spong [32], [44]. We consider the Lyapunov function candidate

$$
V(t)=\frac{1}{2} \mathbf{s}^{T} \mathbf{M} \mathbf{s}+\frac{1}{2} \tilde{\mathbf{x}}^{T}\left(\mathbf{K}_{P}+\mathbf{\Lambda} \mathbf{K}_{D}\right) \tilde{\mathbf{x}}+\frac{1}{2} \tilde{\mathbf{a}}^{T} \Gamma \tilde{\mathbf{a}}
$$

where $\mathbf{s}, \mathbf{M}, \tilde{\mathbf{x}}, \boldsymbol{\Lambda}, \mathbf{K}_{P}, \mathbf{K}_{D}$, and $\boldsymbol{\Gamma}$ are as defined in the previous sections and $\tilde{\mathbf{a}}$ is a parameter estimate error defined as

$$
\tilde{\mathbf{a}}=\hat{\mathbf{a}}-\mathbf{a}
$$

where $\hat{\mathbf{a}}$ is an estimate of the parameters, a, as previously defined. Taking the derivative of (20) along system trajectories yields

$$
\dot{V}(t)=\frac{1}{2} \mathbf{s}^{T} \dot{\mathbf{M}} \mathbf{s}+\mathbf{s}^{T} \mathbf{M} \dot{\mathbf{s}}+\tilde{\mathbf{x}}^{T}\left(\mathbf{K}_{P}+\Lambda \mathbf{K}_{D}\right) \dot{\tilde{\mathbf{x}}}+\tilde{\mathbf{a}}^{T} \Gamma \dot{\tilde{\mathbf{a}}} .
$$

Using the sliding surface $\mathbf{s}$ and reference trajectories $\mathbf{w}$ defined in (5), the system dynamics in (4) can be redefined as

$$
\mathbf{M} \dot{\mathbf{s}}+\mathbf{C s}+\mathbf{Y a}=\mathbf{F}_{r} .
$$

Substituting for Mś from (23) and for a from (21) into (22) gives

$$
\begin{aligned}
\dot{V}(t)=\frac{1}{2} \mathbf{s}^{T} & \dot{\mathbf{M}} \mathbf{s}+\mathbf{s}^{T}\left(\mathbf{F}_{r}-\mathbf{Y} \hat{\mathbf{a}}\right)-\mathbf{s}^{T} \mathbf{C s} \\
& +\tilde{\mathbf{x}}^{T}\left(\mathbf{K}_{P}+\Lambda \mathbf{K}_{D}\right) \dot{\tilde{\mathbf{x}}}+\tilde{\mathbf{a}}^{T}\left(\Gamma \hat{\mathbf{a}}+\mathbf{Y}^{T} \mathbf{s}\right) .
\end{aligned}
$$

Using the fact that $\dot{M}-2 \mathrm{C}$ is skew symmetric we have

$$
\begin{aligned}
\dot{V}(t)=\mathbf{s}^{T}\left(\mathbf{F}_{r}-\mathbf{Y} \hat{\mathbf{a}}\right)+\tilde{\mathbf{x}}^{T}\left(\mathbf{K}_{P}\right. & \left.+\Lambda \mathbf{K}_{D}\right) \dot{\tilde{\mathbf{x}}} \\
& +\tilde{\mathbf{a}}^{T}\left(\boldsymbol{\Gamma} \dot{\hat{\mathbf{a}}}+\mathbf{Y}^{T} \mathbf{s}\right) .
\end{aligned}
$$

Inserting the control law (7) and the modified parameter estimate update law (15) into (25) gives

$$
\dot{V}(t)=-\tilde{\mathbf{x}}^{T} \Lambda \mathbf{K}_{P} \tilde{\mathbf{x}}-\dot{\tilde{\mathbf{x}}}^{T} \mathbf{K}_{D} \dot{\tilde{\mathbf{x}}}-\tilde{\mathbf{a}}^{T} \frac{1}{\tau} \boldsymbol{\Gamma} \mathbf{Y}^{T}\left(\mathbf{Y} \mathbf{Y}^{T}\right)^{-1} \mathbf{Y} \hat{\mathbf{a}} .
$$

Because the last term in (26) may be positive, the system is not asymptotically stable. However, the system is stable in the sense of uniform ultimate boundedness. To determine the stable boundary of the system, we first rewrite (26) as

$$
\dot{V}(t)=-\mathbf{e}^{T} \mathbf{Q e}-\tilde{\mathbf{a}}^{T} \mathbf{B a ̂}
$$


where

$$
\begin{aligned}
\mathbf{e} & =\left[\begin{array}{ll}
\tilde{\mathbf{x}} & \dot{\mathbf{x}}
\end{array}\right]^{T} \\
\mathbf{Q} & =\left[\begin{array}{c:c}
\mathbf{\Lambda} \mathbf{K}_{P} & 0 \\
\hdashline 0 & \mathbf{K}_{D}
\end{array}\right]
\end{aligned}
$$

and

$$
\mathbf{B}=\frac{1}{\tau} \boldsymbol{\Gamma} \mathbf{Y}^{T}\left(\mathbf{Y} \mathbf{Y}^{T}\right)^{-1} \mathbf{Y}
$$

we see now that $\dot{V}(t)<0$ when

$$
\mathbf{e}^{T} \mathbf{Q e}>-\tilde{\mathbf{a}}^{T} \mathbf{B a ̂}
$$

which occurs whenever

$$
\lambda_{\min }(\mathbf{Q})\|\mathbf{e}\|^{2}>\max _{\hat{\mathbf{a}}}\left(-\tilde{\mathbf{a}}^{T} \mathbf{B} \hat{\mathbf{a}}\right)
$$

where $\lambda_{\min }(\mathbf{Q})$ is the minimum eigenvalue of $\mathbf{Q}$. The maximum of $-\tilde{\mathbf{a}}^{T} \mathbf{B} \hat{\mathbf{a}}$ is occurs when

$$
\hat{\mathbf{a}}=\frac{1}{2} \mathbf{a} \text {. }
$$

Substituting (30) into (29) and solving for $\|\mathbf{e}\|$ using the above definition of $\mathbf{B}$ gives

$$
\begin{aligned}
\|\mathbf{e}\| & >\frac{1}{2}\left(\frac{\mathbf{a}^{T} \mathbf{B a}}{\lambda_{\min }(\mathbf{Q})}\right)^{1 / 2} \\
& =\frac{1}{2}\left(\frac{1}{\tau} \frac{\mathbf{a}^{T} \boldsymbol{\Gamma} \mathbf{Y}^{T}\left(\mathbf{Y} \mathbf{Y}^{T}\right)^{-1} \mathbf{Y a}}{\lambda_{\min }(\mathbf{Q})}\right)^{1 / 2} .
\end{aligned}
$$

Equation (31) defines the maximum error for $\dot{V}(t)>0$ and thus represents the boundary of the closed loop system. Larger magnitudes of $\mathbf{e}$ will have $\dot{V}(t)<0$ and the closed-loop human-robot system will therefore converge to this boundary. We see that the magnitude of the error $\mathbf{e}$ will be small when the robot forgetting rate $1 / \tau$ is small. In other words, as $\tau \rightarrow \infty$ the adaptive controller remembers all it has learned about the parameters so that $\mathbf{e} \rightarrow 0$. Because $\lambda_{\max }\left(\boldsymbol{\Gamma} \mathbf{Y}^{T}\left(\mathbf{Y} \mathbf{Y}^{T}\right)^{-1} \mathbf{Y}\right)=\lambda_{\max }(\boldsymbol{\Gamma})$, (31) can be written

$$
\|\mathbf{e}\|>\frac{1}{2}|\mathbf{a}|\left(\frac{1}{\tau} \frac{\lambda_{\max }(\boldsymbol{\Gamma})}{\lambda_{\min }(\mathbf{Q})}\right)^{1 / 2}
$$

which shows that the kinematic tracking error bound is proportional to the actual parameters a of the system.

\section{ACKNOWLEDGMENT}

The authors would like to thank $\mathrm{V}$. Le for programming assistance and also thank S. Cramer, M.D., Ph.D. for medical supervision and helpful comments.

\section{REFERENCES}

[1] W. Rosamond, K. Flegal, G. Friday, K. Furie, A. Go, K. Greenlund, N. Haase, M. Ho, V. Howard, B. Kissela, S. Kittner, D. Lloyd-Jones, M. McDermott, J. Meigs, C. Moy, G. Nichol, C. J. O’Donnell, V. Roger, J. Rumsfeld, P. Sorlie, J. Steinberger, T. Thom, S. Wasserthiel-Smoller, and Y. Hong, "Heart disease and stroke statistics-2007 update: A report from the american heart association statistics committee and stroke statistics subcommittee," Circulation, vol. 115, pp. 69-171, 2007.
[2] B. H. Dobkin, "Strategies for stroke rehabilitation," Lancet Neurol., vol. 3, pp. 528-536, Sep. 2004.

[3] G. Kwakkel, "Impact of intensity of practice after stroke: Issues for consideration," Disability Rehabil., vol. 28, pp. 823-830, 2006.

[4] P. Lum, "Robotic devices for movement therapy after stroke: Current status and challenges to clinical acceptance," Topics Stroke Rehabil., vol. 8, pp. 40-53, 2002.

[5] S. Hesse, H. Schmidt, C. Werner, and A. Bardeleben, "Upper and lower extremity robotic devices for rehabilitation and for studying motor control," Current Opinion Neurol., vol. 16, no. 6, pp. 705-710, 2003.

[6] R. Riener, T. Nef, and G. Colombo, "Robot-aided neurorehabilitation of the upper extremities," Med. Biol. Eng. Comput., vol. 43, pp. 2-10, 2005.

[7] B. T. Volpe, M. Ferraro, D. Lynch, P. Christos, J. Krol, C. Trudell, H. I. Krebs, and N. Hogan, "Robotics and other devices in the treatment of patients recovering from stroke," Current Neurol. Neurosci. Rep., vol. 5, p. 465, 2005.

[8] P. Lum, C. Burgar, M. V. d. Loos, P. C. Shor, O. T. R. M. Majmundar, and R. Yap, "MIME robotic device for upper-limb neurorehabilitation in subacute stroke subjects: A follow-up study," J. Rehabil. Res. Development, vol. 43, pp. 631-642, 2006.

[9] J. L. Emken and D. J. Reinkensmeyer, "Human-robot cooperative movement training: Learning a novel sensory motor transformation during walking with robotic assistance-as-needed," J. Neuroeng. Rehabil., 2007.

[10] J. L. Emken and D. J. Reinkensmeyer, "Robot-enhanced motor learning: Accelerating internal model formation during locomotion by transient dynamic amplification," IEEE Trans. Neural Syst. Rehabil. Eng., vol. 13, pp. 33-39, 2005.

[11] J. L. Patton, M. E. Stoykov, M. Kovic, and F. A. Mussa-Ivaldi, "Evaluation of robotic training forces that either enhance or reduce error in chronic hemiparetic stroke survivors," Exp. Brain Res., vol. 168, pp. 368-383, 2006.

[12] N. Hogan, H. I. Krebs, B. Rohrer, J. J. Palazzolo, L. Dipietro, S. E. Fasoli, J. Stein, R. Hughes, W. R. Frontera, D. Lynch, and B. T. Volpe, "Motions or muscles? Some behavioral factors underlying robotic assistance of motor recovery," J. Rehabilitation Res. Dev., vol. 43, pp. 605-618, Sep./Oct. 2006.

[13] H. I. Krebs, N. Hogan, M. L. Aisen, and B. T. Volpe, "Robot-aided neurorehabilitation," IEEE Trans. Rehabil. Eng., vol. 6, no. 1, pp. 75-87, Mar. 1998

[14] R. A. Schmidt, Motor Control and Learning: A Behavioral Emphasis. Champaign, IL: Human Kinetics, 2005.

[15] H. I. Krebs, B. T. Volpe, D. Lynch, and N. Hogan, "Stroke rehabilitation: An argument in favor of a robotic gym," in 2005 IEEE 9th Int. Conf. Rehabil. Robot., Chicago, IL, 2005, pp. 219-222.

[16] T. Nef, M. Mihelj, and R. Riener, "ARMin: A robot for patient-cooperative arm therapy," Med. Biol. Eng. Comput., vol. 45, pp. 887-900, 2007.

[17] J. He, E. J. Koeneman, R. S. Schultz, D. E. Herring, J. Wanberg, H. Huang, T. Sugar, R. Herman, and J. B. Koeneman, "RUPERT: A device for robotic upper extremity repetitive therapy," in IEEE-EMBS 27th Annu. Int. Conf. Eng. Medi. Biol. Soc., 2005, pp. 6844-6847.

[18] D. J. Reinkensmeyer, L. E. Kahn, M. Averbuch, A. McKenna-Cole, B. D. Schmit, and W. Z. Rymer, "Understanding and treating arm movement impairment after chronic brain injury: Progress with the arm guide," J. Rehabil. Res. Develop., vol. 37, pp. 653-662, 2000.

[19] C. D. Takahashi, L. Der-Yeghiaian, V. H. Le, and S. C. Cramer, "A robotic device for hand motor therapy after stroke," rehabilitation robotics, 2005," in ICORR 2005. 9th Int. Conf., 2005, pp. 17-20.

[20] R. Colombo, F. Pisano, S. Micera, A. Mazzone, C. Delconte, M. C. Carrozza, P. Dario, and G. Minuco, "Robotic techniques for upper limb evaluation and rehabilitation of stroke patients," neural systems and rehabilitation engineering," IEEE Trans. Neural Syst. Rehabil. Eng., vol. 13, no. 3, pp. 311-324, Sep. 2005.

[21] L. L. Cai, A. J. Fong, C. K. Otoshi, Y. Liang, J. W. Burdick, R. R. Roy, and V. R. Edgerton, "Implications of assist-as-needed robotic step training after a complete spinal cord injury on intrinsic strategies of motor learning.," J. Neurosci., vol. 26, pp. 10564-10568, 2006.

[22] J. F. Israel, D. D. Campbell, J. H. Kahn, and T. G. Hornby, "Metabolic costs and muscle activity patterns during robotic- and therapist-assisted treadmill walking in individuals with incomplete spinal cord injury," Phys. Therapy, vol. 86, pp. 1466-1478, Nov. 2006.

[23] H. I. Krebs, J. J. Palazzolo, L. Dipietro, M. Ferraro, J. Krol, K. Rannekleiv, B. T. Volpe, and N. Hogan, "Rehabilitation robotics: Performance-Based progressive robot-assisted therapy," Auton. Robot, vol. 15, no. 1, pp. 7-20, 2003. 
[24] L. E. Kahn, W. Z. Rymer, and D. J. Reinkensmeyer, "Adaptive assistance for guided force training in chronic stroke," in Proc. 26th EMBC Annu. Int. Conf. Eng. Medicine Biol. Soc., San Francisco, CA, 2004, vol. 1, pp. 2722-2725.

[25] P. S. Lum, C. G. Burgar, P. C. Shor, M. Majmundar, and M. V. d. Loos, "Robot-assisted movement training compared with conventional therapy techniques for the rehabilitation of upper limb motor function following stroke," Arch. Phys. Med. Rehab. , vol. 83, pp. 952-959, 2002.

[26] D. J. Reinkensmeyer, J. L. Emken, and S. C. Cramer, "Robotics, motor learning, and neurologic recovery," Annu. Rev. Biomed. Eng., vol. 6, pp. 497-525, 2004.

[27] R. J. Sanchez, E. Wolbrecht, R. Smith, J. Liu, S. Rao, S. Cramer, T. Rahman, J. E. Bobrow, and D. J. Reinkensmeyer, "A pneumatic robot for re-training arm movement after stroke: Rationale and mechanical design," in Proc. IEEE Int. Conf. Rehab. Robot., 2005, pp. 500-504.

[28] E. T. Wolbrecht, J. Leavitt, D. J. Reinkensmeyer, and J. E. Bobrow, "Control of a pneumatic orthosis for upper extremity stroke rehabilitation," in 28th Annu. Int. Conf. IEEE Eng. Medicine Biol. Soc. , New York, 28th, 2006, pp. 2687-2693.

[29] E. T. Wolbrecht, Adaptive, assist-as-needed control of a pneumatic orthosis for optimizing robotic movement training following stroke Dept. Mechan. Aerospace Eng., Univ. California, Irvine, 2007.

[30] D. J. Reinkensmeyer, E. T. Wolbrecht, and J. Bobrow, "A computational model of human-robot load sharing during robot-assisted arm movement training after stroke," presented at the 28th Annu. Int. Conf. IEEE Eng. Medicine Biol. Soc., Lyon, France, 2007.

[31] T. Rahman, W. Sample, and R. Seliktar, "Design and testing of WREX," in Advances in Rehabilitation Robotics. New York: Springer, 2004, pp. 243-250.

[32] J. J. E. Slotine and W. Li, Applied Nonlinear Control. Upper Saddle River, NJ: Prentice-Hall, 1991.

[33] D. Erol, V. Mallapragada, N. Sarkar, G. Uswatte, and E. Taub, "A new control approach to robot assisted rehabilitation," in 2005 IEEE 9th Int. Conf. Rehabil. Robotics, Chicago, IL, 2005, pp. 323-328.

[34] L. E. Kahn, M. L. Zygman, W. Z. Rymer, and D. J. Reinkensmeyer, "Robot-assisted reaching exercise promotes arm movement recovery in chronic hemiparetic stroke: A randomized controlled pilot study," $J$. Neuroeng. Rehabil., vol. 3, p. 12, 2006.

[35] N. Hogan, "Interactive robots for neuro-rehabilitation," Restor. Neurol. Neurosci., vol. 22, no. 3-5, pp. 349-358, 2004.

[36] E. Todorov, "Optimality principles in sensorimotor control (review)," Nature Neurosci., pp. 907-915, 2004.

[37] J. L. Emken, R. Benitez, A. Sideris, J. E. Bobrow, and D. J. Reinkensmeyer, "Motor adaptation as a greedy optimization of error and effort," J. Neurophysiol., pp. 3997-4006, Mar. 2007.

[38] T. Nef and R. Riener, "ARMin-Design of a novel arm rehabilitation robot," in Proc. IEEE 9th Int. Conf. Rehabil. Robotics, 2005, pp. 57-60.

[39] M. Mihelj, T. Nef, and R. Riener, "A novel paradigm for patient-cooperative control of upper-limb rehabilitation robots," $A d v$. Robotics, vol. 21, pp. 843-867, 2007.

[40] A. Montagner, A. Frisoli, L. Borelli, C. Procopio, M. Bergamasco, M. Carboncini, and B. Rossi, "A pilot clinical study on robotic assisted rehabilitation in VR with an arm exoskeleton device," presented at the Virutal Rehabil. Conf., Venice, Italy, 2007.

[41] J. L. Emken, J. Beres-Jones, S. J. Harkem, C. Ferreira, and D. J. Reinkensmeyer, "Feasibility of manual teach-and-replay and continuous impedance shaping for robotic locomotor training following spinal cord injury," IEEE Trans. Biomed. Eng., vol. 55, no. 1, pp. 322-334, Jan. 2008.

[42] R. Riener, L. Lunenburger, S. Jezernik, M. Anderschitz, G. Colombo, and V. Dietz, "Patient-cooperative strategies for robot-aided treadmill training: First experimental results," IEEE Trans. Neural Syst. Rehabil. Eng., vol. 13, no. 3, pp. 380-394, Sep. 2005.
[43] D. Aoyagi, W. E. Ichinose, S. J. Harkema, D. J. Reinkensmeyer, and J. E. Bobrow, "A robot and control algorithm that can synchronously assist in naturalistic motion during body-weight-supported gait training following neurologic injury," IEEE Trans. Neural Syst. Rehabil. Eng., vol. 15 , no. 3, pp. 387-400, Sep. 2007.

[44] M. W. Spong and M. Midyasagar, Robot Dynamics and Control. New York: Wiley, 1989.

Eric T. Wolbrecht (M'06) received the B.S. degree in mechanical engineering from Valparaiso University, Valparaiso, IN, in 1996, the M.S. degree in mechanical engineering from Oregon State University, Corvallis, in 1998, and the Ph.D. degree in mechanical and aerospace engineering from the University of California, Irvine, in 2007.

He is currently an Assistant Professor in the Department of Mechanical Engineering at the University of Idaho, Moscow. His research interests include robotics, nonlinear and adaptive control, compliant actuation, motor learning, and neurorehabilitation.

Dr. Wolbrecht is a member of the American Society of Mechanical Engineering.

Vicky Chan received the B.S. degree in human biology (Magna Cum Laude) and the M.S. degree in physical therapy from Texas Woman's University, Denton.

She worked at Kindred Hospital, Dallas, TX, for four years treating mostly ventilator dependent patients. Currently, she is the Primary Physical Therapist for the Acute Rehabilitation Unit at the University of California, Irvine.

David J. Reinkensmeyer (S'93-M'93) received the B.S. degree in electrical engineering from the Massachusetts Institute of Technology, Cambridge, in 1988, and the M.S. and Ph.D. degrees in electrical engineering from the University of California, Berkeley, in 1991 and 1993, respectively.

He was a Research Associate at the Rehabilitation Institute of Chicago and Northwestern University Medical School from 1994 to 1997. He is currently a Professor in the Department of Mechanical and Aerospace Engineering and the Department of Biomedical Engineering at the University of California, Irvine. His research interests are in neuromuscular control, motor learning, robotics, and neurorehabilitation.

Dr. Reinkensmeyer is an Associate Editor of the IEEE TRANSACTIONS ON NEURAL SYSTEMS AND REHABILITATION ENGINEERING.

James E. Bobrow (M'84) received the Ph.D. degree from the University of California, Los Angeles, in 1983.

After graduate school, he was a Senior Programmer Analyst at McDonnell Douglas Automation Company, where he developed CAM software for the Unigraphics system. He joined the University of California, Irvine (UCI), as an Assistant Professor in 1984. He is currently a Professor of Mechanical and Aerospace Engineering at UCI. While at UCI, his research interests have included optimal control and motion planning for robots, design of pneumatic actuators and sensors for automation systems, robots for rehabilitation, and machine learning systems. He has also been a Visiting Professor in Computer Science at Stanford University, Stanford, CA, and in Mechanical Engineering at the Massachusetts Institute of Technology, Cambridge, and he has created robots and automation devices for several startup companies, including Robomedica, Inc., Irvine, CA, and Cobra Technologies, Smyrna, GA. He has served on the program committees or organizing committees of the leading conferences in control systems and robotics. He is currently on the Engineering steering committee for Robomedica, Inc.

Dr. Bobrow is an Associate Editor of the IEEE TRANSACTIONS ON SYSTEMS, MAN, AND CYBERNETICS-PART B: CYBERNETICS. 